\title{
Covid-19: come la pandemia ha aumentato la food insecurity e alterato il regime alimentare nelle famiglie italiane
}

\author{
ARIANNA DONDI', EGIDIO CANDELA², FRANCESCA MORIGI ${ }^{2}$, JACOPO LENZI ${ }^{3}$, MARCELLO LANARI' \\ 'UOC di Pediatria d'Urgenza, Pronto Soccorso e Osservazione Breve Intensiva, IRCCS - AOU di Bologna \\ ${ }^{2}$ Scuola di Specializzazione in Pediatria - Alma Mater Studiorum, Università di Bologna, Italia \\ ${ }^{3}$ Dipartimento di Scienze Biomediche e Neuromotorie, Alma Mater Studiorum, Università di Bologna
}

La pandemia da SARS-CoV-2 e le conseguenti misure di contenimento stanno causando un aumento della insicurezza alimentare (food insecurity) in tutto il mondo. Alla food insecurity sono associate numerose implicazioni negative che interessano in particolare i gruppi più vulnerabili, in modo particolare i bambini e adolescenti. Poiché le restrizioni correlate alla pandemia sono ancora in atto e gli effetti si vedranno ulteriormente a distanza, è fondamentale che i programmi di supporto socio-sanitari si ispirino sempre più a concetti di uguaglianza e sostenibilità, per assicurare la possibilità di procurarsi cibo adeguato, per quantità e qualità. Ci riguarda.

\section{FOOD INSECURITY: L'IMPORTANZA DELL'ALIMENTAZIONE}

L'insicurezza alimentare, chiamata con terminologia anglosassone food insecurity (FI), definisce la mancanza di accesso in qualsiasi momento a cibo sufficiente da un punto di vista della quantità e della qualità per una vita attiva e sana ${ }^{1}$. La malnutrizione, sia in termini di sottonutrizione per scarsità di cibo, che di ipernutrizione per assunzione prevalente di alimenti ipercalorici ricchi di zuccheri e grassi, è un problema rilevante che può minare la salute dei bambini e il loro svilup$\mathrm{po}^{2,3}$. La prevalenza della FI è aumentata negli ultimi anni in diversi Paesi europe $^{46}$, fenomeno osservato anche in Italia, dove la FI è passata dal $7 \%$ del 2009 al 17\% del $2012^{7}$.

\section{FOOD INSECURITY E COVID: COSA Ė CAMBIATO?}

La pandemia da sindrome respiratoria acuta severa da coronavirus- 2 (SARS-CoV-2) e tutte le misure di contenimento attuate per rallentarne la diffusione ${ }^{8}$ hanno causato una delle

\section{COVID-19: HOW THE PANDEMIC HAS INCREASED FOOD INSECURITY} AND ALTERED THE ITALIAN FAMILIES' DIET

(Medico e Bambino 2021;40(5):315-320. doi: 10.53126/MEB40315)

\section{Key words}

Covid-19, Food insecurity, Social determinants of health, Body weight, Junk food, Family, Pandemic

\section{Summary}

Background - The Severe Acute Respiratory Syndrome Coronavirus-2 (SARS-CoV-2) pandemic and subsequent containment measures are causing a worldwide increase in food insecurity (FI). Food insecurity has been related to a number of negative health implications, particularly for the most vulnerable population, including children and adolescents.

Aim - To investigate the effects on Fl during the lockdown in ltaly and the changes in eating habits and body weight in the paediatric population 6 months after the beginning of the pandemic.

Materials and Methods - An online anonymous cross-sectional survey was elaborated and proposed to the parents of children $<18$ years old.

Results $-8.3 \%$ reported that their families were at risk of $\mathrm{Fl}$ before the outbreak of SARS$\mathrm{CoV}-2$ pandemic. This percentage dramatically doubled after the pandemic began. Moreover, $27.3 \%$ of the parents reported that their children were eating more, with a concomitant increase in "junk food" consumption. About one third of respondents declared an increase in children's weight, while weight loss were uncommon and prevailed among adolescents. It was observed that parents' higher age, higher parents' school grade and working parents were protective factors against FI.

Conclusion - Since the SARS-CoV-2 pandemic is requiring restrictions again, it is crucial that health care and social protection programmes take into account concepts of equity and sustainability to ensure adequate food and nutrition security for everyone.

crisi più gravi dell'economia mondiale dalla fine della Seconda Guerra Mondiale a oggi ${ }^{9}$. La maggior parte dei Paesi ne sta affrontando le drammati- che conseguenze sociali ed economiche già da alcuni mesi.

Di fatto, la pandemia ha colpito i sistemi alimentari sia direttamente, in- 
fluenzando la disponibilità e la domanda di cibo, sia indirettamente, riducendo la capacità di produrre e distribuire alimenti ${ }^{10}$. Perdita del posto di lavoro e mancanza di sostegno sociale sono fattori di rischio per una più elevata probabilità di FI e sono stati esasperati dalla pandemia da SARS-CoV$2^{11}$. La chiusura delle scuole per lunghi periodi ha inoltre comportato per molti ragazzi anche il venir meno di un pasto al giorno sicuro, controllato e di buona qualità ${ }^{12}$.

È ipotizzabile inoltre che un livello elevato di FI possa favorire la diffusione dell'epidemia ${ }^{13}$; è noto infatti come la malnutrizione possa ridurre la funzionalità del sistema immunitario ${ }^{14}$ potendo rendere la malattia Covid-19 più severa e maggiormente diffusibi$\mathrm{l}^{15}$. A conferma di ciò, la malnutrizione e l'obesità sono state correlate con un aumento dei tassi di ospedalizzazione per Covid-19 nelle popolazioni adulte degli Stati Uniti ${ }^{16}$. Allo stesso modo, la paura e l'incertezza correlate alla pandemia sono fattori di stress che possono portare a un eccessivo consumo di cibo ${ }^{17}$, spesso di cattiva qualità, con le possibili note conseguenze metaboliche.

\section{LA NOSTRA INDAGINE}

Abbiamo deciso di condurre uno studio per indagare gli effetti del lockdown su FI, abitudini alimentari e conseguente variazione di peso nella popolazione pediatrica italiana, 6 mesi dopo l'inizio della pandemia.

Con questo scopo abbiamo costruito un'indagine online costituita da 78 domande e l'abbiamo proposta a genitori con figli minorenni residenti in Italia. Per l'analisi dei risultati sono stati utilizzati indici statistici descrittivi e inferenziali.

Il questionario si poneva l'obiettivo di analizzare anche la correlazione fra la FI e alcuni dei determinanti sociali di salute. Fra questi vi erano la condizione abitativa, la composizione della famiglia, il livello di scolarizzazione e l'occupazione dei genitori (tipo di occupazione, impegno nell'orario di lavoro e cambiamenti nelle condizioni

\section{Box 1 - I DETERMINANTI dI SALUTE: LA CAUSA DELlE CAUSE}

I determinanti di salute costituiscono i fattori che influenzano lo stato di salute di un individuo e - più estesamente - di una comunità o di una popolazione. Fra la metà degli anni '60 e inizi anni '70 si è fatta progressivamente strada la consapevolezza che un approccio esclusivamente di tipo biomedico fosse insufficiente a soddisfare i bisogni di salute della gran parte della popolazione a livello mondiale, principalmente per quanto riguarda $i$ più poveri e svantaggiati ${ }^{18}$.

L'analisi del perché l'aspettativa di vita per un vomo contemporaneo appartenente alla parte povera della città di Baltimora (Stati Uniti) fosse di circa 63 anni, mentre quella di un suo coetaneo della parte benestante di 83 , ha fatto emergere un nuovo modo di pensare alla salute e alla malattia. Lo stesso divario di 20 anni di aspettativa di vita è infatti presente fra una donna nata in India e una negli Stati Uniti ${ }^{19}$.

E oggi assodato, grazie a numerose pubblicazioni scientifiche, come le condizioni di svantaggio sociale costituiscano un fattore di rischio centrale per la salute. Tali studi, a partire dal primo lavoro della Commissione sui Determinanti Sociali dell'Organizzazione Mondiale della Sanità (2005-2008), sottolineano come il valore dell'appartenenza a contesti di vita differenti distribuisca in maniera diversa salute e malattia nella popolazione. I determinanti sociali di salute costituirebbero, in particolare, i principali responsabili di simili processi: la cosiddetta "causa delle cause" della salute, sia fisica che mentale. Questi elementi sono aggregabili in varie categorie: livello di istruzione; reti sociali e comunitarie; ambienti di vita e lavoro; comportamenti personali e stili di vita; accesso ai servizi sanitari; condizioni socio-economiche, abitative, culturali, politiche e ambientali.

Le persone appartenenti a classi sociali più fragili risultano pertanto più esposte, nei propri contesti di vita e lungo l'arco della propria esistenza, agli effetti sfavorevoli dei fattori di rischio (materiali, psicosociali, comportamentali) per la salute, meno dotate dell'insieme di fattori in grado di proteggerle da eventi avversi e infine più danneggiate dalle conseguenze sociali delle patologie ${ }^{20}$

La disuguaglianza in salute si traduce anche oggi in Italia in un impatto nell'aspettativa di vita nelle nostre città. Ė suggestivo lo "spunto per leggere i bisogni e i risultati delle politiche" di Costa e coll. A Torino una persona che utilizza il tram che attraversa la città, dalla collina alto-borghese all'estremo Est, per giungere nella barriera operaia dell'estremo Nord-Ovest, vede salire a bordo dei passeggeri che perdono cinque mesi di speranza di vita ogni chilometro che il mezzo percorre: alla fine del percorso un divario di più di quattro anni di aspettativa di vita separerà i benestanti della collina dagli abitanti degli isolati più poveri ${ }^{21}$.

\section{BOX 2 - HUNGER VITAL SIGN}

Negli ultimi 12 mesi ci siamo preoccupati della possibilità che il nostro cibo finisse prima di avere i soldi per comprarne dell'altro (è capitato spesso / è capitato alcune volte / non è capitato mai).

Negli ultimi 12 mesi il cibo che abbiamo comprato non è stato sufficiente e non avevamo denaro per comprarne di più (è capitato spesso / è capitato alcune volte / non è capitato mai).

di lavoro durante la pandemia), la difficoltà economica, lo stress percepito dai figli.

Il questionario si poneva l'obiettivo di analizzare anche la correlazione fra la FI e alcuni dei determinanti sociali di salute $(B o x 1)^{18221}$.

La FI è stata indagata attraverso un questionario di screening rapido, denominato Hunger Vital Sign (HVS), sviluppato nel 2010 dal team di Children's Health Watch, costituito da solo due domande in grado di identificare il rischio di FI nella popolazione pediatrica e pertanto adatto ai fini del nostro studio $\left(\right.$ Box 2) ${ }^{22}$.
In prima istanza, il questionario era stato validato come uno strumento di screening costituito da soli due quesiti per poter rilevare in modo rapido la FI in famiglie con bambini di età inferiore a 3 anni. Hager e coll. ${ }^{22}$ avevano riscontrato come una risposta positiva a entrambe le domande conferisse al test una sensibilità del $97,0 \%$ e una specificità dell' $83,0 \%$ rispetto alla scala di sicurezza alimentare in uso nelle famiglie statunitensi, costituita da 18 quesiti. L'HVS è stato successivamente validato come screening per la FI per bambini, adolescenti e adulti. 


\begin{tabular}{|c|c|c|c|}
\hline \multicolumn{4}{|c|}{ DESCRIZIONE DELLA POPOLAZIONE DELLO STUDIO } \\
\hline CARATTERISTICA & $\%$ & CARATTERISTICA & $\%$ \\
\hline Età [anni] & & Smart working per almeno un genitore & \\
\hline$\leq 30$ & 7,6 & No & 33,2 \\
\hline $\begin{array}{l}31-35 \\
36-40\end{array}$ & 16,4 & Sì & 66,8 \\
\hline $36-40$ & 28,6 & Cassa integrazione per almeno un genitore & \\
\hline $41-45$ & 26,0 & No & 61,9 \\
\hline $46-50$ & 15,7 & Sì & 38,1 \\
\hline & & Situazione economica della famiglia dopo la pandemia & \\
\hline Paese di origine & & Migliorata & 2,6 \\
\hline Italia & 96,7 & Invariata & 54,6 \\
\hline Non Italia & 3,3 & Lievemente peggiorata & 37,4 \\
\hline Area di residenza & & Peggiorata & 4,7 \\
\hline Nord Italia & 88,5 & Divenuta critica & 0,7 \\
\hline Centro Italia & 6,1 & Almeno un genitore ha perso il lavoro & \\
\hline Sud Italia & 5,4 & No & 89,1 \\
\hline Grado di istruzione del genitore compilante & & Sì & 10,9 \\
\hline Scuola media o inferiore & 5,4 & Come immagina il futuro economico della famiglia? & \\
\hline $\begin{array}{l}\text { Scuola superiore } \\
\text { Laurea o titolo superiore }\end{array}$ & 36,8 & Migliore & 2,3 \\
\hline Laurea o titolo superiore & 58,8 & Invariato & 39,9 \\
\hline Grado di istruzione dell'altro genitore & & Più difficile & 50,5 \\
\hline Scuola media o inferiore & 15,5 & Molto più difficile & 6,1 \\
\hline Scuola superiore & 44,8 & Seriamente a rischio & 1,2 \\
\hline Laurea o titolo superiore & 39,7 & Cosa è mancato di più ai suoi figli?** & \\
\hline Condizione lavorativa dei genitori & & Andare a scuola & 30,6 \\
\hline Entrambi disoccupati & 0,8 & Uscire di casa & 46,9 \\
\hline Uno occupato & 15,6 & Incontrare gli amici & 80,2 \\
\hline Entrambi occupati & 83,7 & Incontrare i parenti & 41,4 \\
\hline Situazione economica della famiglia & & Praticare sport & 42,3 \\
\hline Agiata & 39,1 & Ha notato un cambiamento di umore nei suoi figli? & \\
\hline Qualche difficoltà ma globalmente soddisfacente & 53,7 & No & 22,7 \\
\hline Parecchie difficoltà & 6,7 & Sì, più nervosi, preoccupati o tristi & 74,7 \\
\hline Spesso insostenibile & 0,5 & Sì, il loro umore è migliorato & 2,6 \\
\hline Numero di bambini nella famiglia & & I suoi figli hanno provato sentimenti di solitudine? & \\
\hline 1 & 41,3 & & \\
\hline 2 & 48,5 & Sì, ma non l'hanno mai verbalizzato & 31,4 \\
\hline 3 & 7,8 & Sì, l'hanno verbalizzato & 36,1 \\
\hline$>3$ & 2,4 & & \\
\hline
\end{tabular}

Tabella I. *Possibili più risposte.

\section{LA POPOLAZIONE}

Il questionario è stato compilato da 7958 genitori. La Tabella I riporta la descrizione della popolazione dello studio.

La maggioranza degli intervistati era di sesso femminile $(91,3 \%)$, italiana $(96,7 \%)$ e residente nel Nord Italia (88,5\%). La maggior parte aveva un grado di istruzione pari a scuola superiore o Università $(95,6 \%)$. Nell' $83,7 \%$ delle famiglie entrambi i genitori erano impiegati e più del $90 \%$ ha descritto la propria situazione economica come "agiata" o "nel complesso soddisfacente". Circa il 40\% dei partecipanti ha riferito che, dall'inizio della pandemia, la propria situazione economica era in qualche modo peggiorata e più del
$50 \%$ ha definito il futuro economico della famiglia "più difficile" o "a rischio". Nel 10,9\% dei casi almeno un genitore aveva perso il lavoro dall'inizio della pandemia. Per quanto riguarda le esperienze e l'attitudine rispetto al lockdown, i genitori ritenevano che $\mathrm{i}$ loro figli avessero sofferto soprattutto per la mancanza degli amici e riportavano che questi erano diventati più ansiosi e si sentivano soli nel $74,7 \%$ e nel $67,5 \%$ dei casi, rispettivamente.

\section{DRAMMATICI EFFETTI DEL LOCKDOWN: FOOD INSECURITY}

L'analisi della FI e delle abitudini alimentari ha incluso 6094 risposte. L'andamento della FI prima e dopo l'i- nizio della pandemia è illustrato nelle Figure 1 e 2. Abbiamo riscontrato che la frequenza con cui i genitori si preoccupavano delle loro scorte alimentari è cambiata significativamente dopo la pandemia $(\mathrm{p}<0,001)$. Più specificamente, la preoccupazione era peggiorata in 654 genitori $(10,7 \%)$ e migliorata in $93(1,5 \%)$. Analogamente, 150 famiglie $(2,5 \%)$ dichiaravano di essere rimaste senza cibo più spesso rispetto il periodo antecedente la pandemia.

I fattori di rischio e quelli protettivi per un peggioramento significativo della FI sono elencati nella Tabella II.

Inoltre, è stata documentata una significativa associazione tra famiglie che non avevano sufficiente cibo a disposizione e incremento del nervosismo dei bambini. 


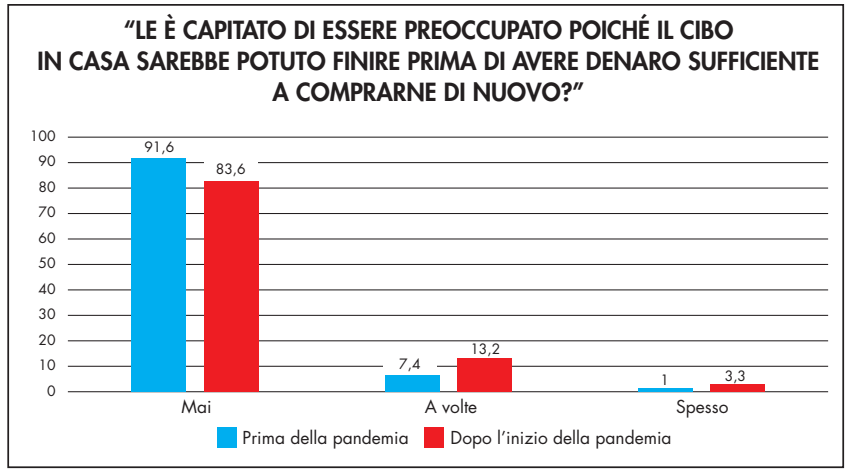

Figura 1. Frequenza con cui i genitori si sono preoccupati di esaurire il cibo, nei 12 mesi precedenti e nei 6 mesi successivi l'inizio della pandemia da Covid-19.

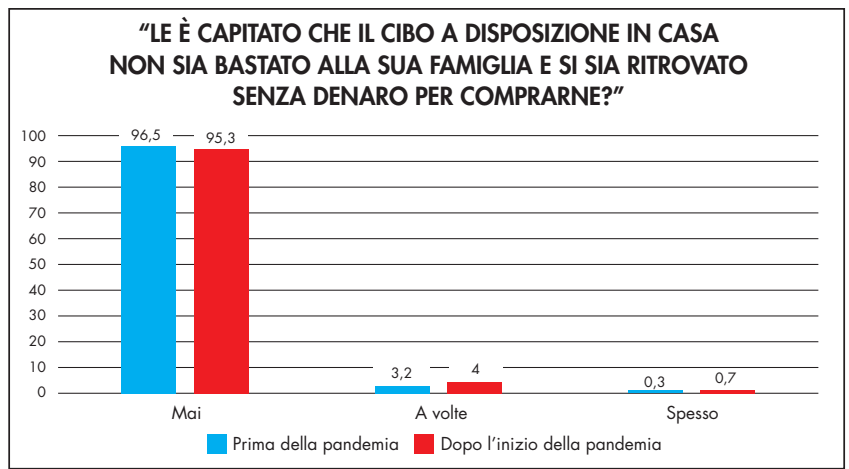

Figura 2. Frequenza con cui i genitori avevano finito il cibo e non avevano abbastanza soldi per comprare altro, prima e dopo l'inizio della pandemia da Covid-19.

\section{FATTORI DI RISCHIO E DI PROTEZIONE PER UN PEGCIORAMENTO DELLA FOOD INSECURITY}

Fattori di rischio
- Situazione economica difficile o insostenibile
- Avere più di un figlio
- Almeno un genitore in cassa integrazione
- Peggioramento della situazione economica
- Residenza nel Sud Italia
- Visione negativa del proprio futuro economico

Tabella II

\section{EFFETTI DEL LOCKDOWN SU ALIMENTAZIONE E PESO}

Globalmente, il 40,4\% dei genitori ha riportato un cambiamento nell'assunzione di cibo da parte dei figli. Tra le 1679 famiglie che hanno dichiarato un aumento dell'apporto alimentare (27,5\% del campione totale) è stato osservato un incremento del consumo di snack e merendine nel $60 \%$ dei casi, succhi di frutta industriali nel $14 \%$ e bevande analcoliche zuccherate nel $10,4 \%$. Il $32,1 \%$ delle famiglie ha inoltre riportato un aumento ponderale dei propri figli, mentre il 6,5\% un calo (Figura 3).

In generale, nella nostra analisi, un aumento del consumo alimentare è stato associato a una maggiore preoccupazione dei genitori per l'esaurimento del cibo e a una situazione economica difficile o insostenibile. Altri fattori di rischio sono risultati l'avere più di un bambino, figli di età superiore ai 2 anni o che avvertivano la mancanza nel praticare un'attività sportiva $o$ all'aperto. $\mathrm{Al}$ contrario, una situazione economica complessivamente soddisfacente è stata associata a un con- sumo di cibo più regolare da parte dei bambini.

$\mathrm{E}$ interessante notare come sia un miglioramento, sia un peggioramento dell'umore è stato associato a una modifica dell'alimentazione, in termini di aumento o di diminuzione. Ove presente, la perdita di peso è risultata invece significativamente correlata all'età adolescenziale.

È molto probabile che, a seguito della pandemia da SARS-CoV-2, si assista a un marcato aumento della FI a livello mondiale, in particolar modo nelle popolazioni più vulnerabili e ancor più in quelle con prole di minore età $^{23,24}$. Sulla base dei primi dati rilevati dal World Food Program e dalla United Nations Food and Agriculture (FAO), la pandemia potrebbe infatti almeno duplicare il numero di persone che soffrono di $\mathrm{FI}^{25}$.

La maggior parte dei partecipanti al nostro questionario sono residenti nel Nord Italia, in particolare nelle re-

\section{COSA ABBIAMO IMPARATO}

gioni (Emilia-Romagna, Lombardia e Veneto) dove la prima ondata di infezione è stata particolarmente massiva e severa. É importante notare come questa area geografica sia riconosciuta come una delle più ricche e benestanti in Europa ${ }^{26}$ e dove il livello di assistenza socio-sanitaria è ritenuto da sempre solido ed efficiente ${ }^{27}$.

Dal nostro questionario emerge che la FI è aumentata in maniera drammatica fino a raddoppiare la sua prevalenza a distanza di 6 mesi dalla diffusione del virus. È inoltre allarmante l'aumento significativo di famiglie preoccupate per il potenziale esaurimento di scorte alimentari, o che abbiano effettivamente sperimentato un concreto depauperamento della capacità di procurarsi alimenti. Queste ultime sono risultate per lo più famiglie già in precedenza soggette a instabilità economica per le quali le misure di contenimento della pandemia hanno causato un drammatico peggioramento di tale condizione.

I fattori di protezione (Tabella II) che abbiamo documentato sono identificati, comprensibilmente, come protettivi per la FI anche in studi precedenti la pandemia ${ }^{28,29}$. I fattori di rischio per un incremento della FI non sono peraltro sorprendenti, poiché ricollegabili a una minore disponibilità economica: fra questi, si rileva come famiglie residenti nel Sud Italia, generalmente con redditi familiari minori rispetto al Nord Italia ${ }^{30}$, siano a maggior rischio. $\mathrm{E}$ inoltre noto come $\mathrm{i}$ soggetti migranti siano maggiormente esposti per ragioni varie alla $\mathrm{FI}^{31}$. 


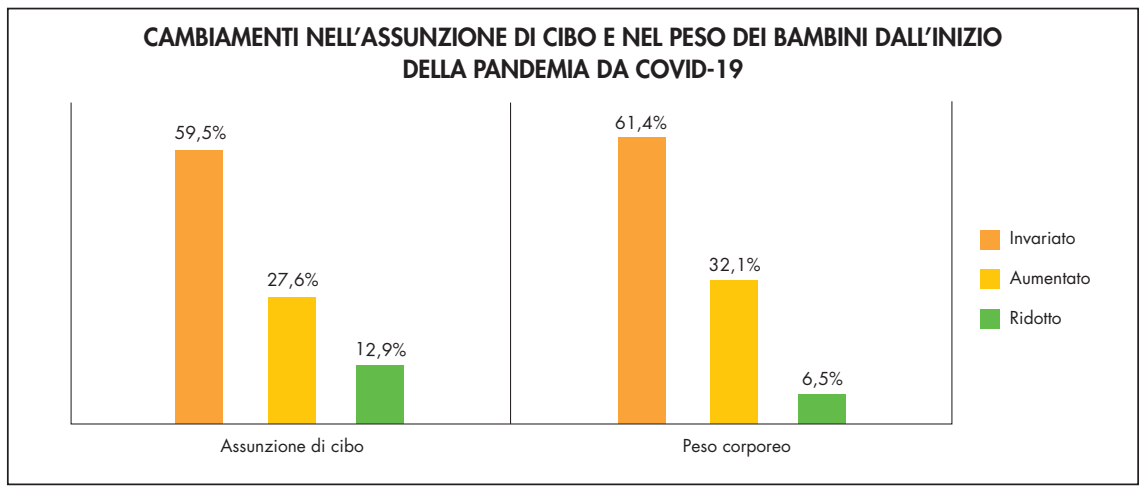

Figura 3

Tuttavia tale popolazione rappresenta nel nostro sondaggio soltanto il $3 \%$ del totale: presumibilmente per tale motivo non abbiamo rilevato in questo sottogruppo un incremento sostanziale della FI. Va comunque considerato come anche uno studio condotto negli Stati Uniti ha rilevato che i migranti non siano stati maggiormente colpiti dalla FI nel periodo della pandemia ${ }^{32}$.

Un altro effetto delle misure di contenimento da tenere in considerazione rilevato nel nostro campione è stato l'aumento dell'apporto alimentare e del consumo del cosiddetto cibo spazzatura (junk food). Le nostre osservazioni sono state confermate anche da altri studi che hanno analizzato le modifiche alimentari durante la pandemia, sia in Italia che negli Stati Uni$\mathrm{ti}^{33,34}$. Ci sembra importante rilevare come i bambini a cui sono mancate maggiormente le attività all'aria aperta abbiano consumato più cibo.

Un aumento di peso è stato osservato nel $32 \%$ dei casi, come peraltro riportato anche in altri studi effettuati su popolazioni pediatriche e adulte ${ }^{35-39}$. $\dot{\mathrm{E}}$ noto che lo stress può stimolare il desiderio di assumere cibo, anche senza la percezione del senso di fame ${ }^{40}$. Il lockdown, tra le varie limitazioni, ha impedito ai bambini di svolgere attività all'aperto e di andare a scuola, rendendo oltremodo difficile il mantenimento di uno stile di vita sano e un fisiologico equilibrio tra dispendio e assunzione di calorie. È stato ipotizzato che lo stress causato dalla pandemia abbia slatentizzato dei sintomi depressivi latenti che hanno poi portato a comportamenti anomali rispetto al cibo (sovra- nutrizione oppure, all'opposto, condotte restrittive) ${ }^{41}$. Per converso, abbiamo evidenziato come a rischio di dimagrimento fossero gli adolescenti sopra i 14 anni: in effetti sappiamo come l'importanza della forma fisica e il peso siano strettamente correlati a problematiche emotive in questa fascia di età ${ }^{42}$. In periodi stressanti come quello che stiamo vivendo, riteniamo che monitorare queste manifestazioni negli adolescenti sia di grande importanza.

\section{LAVORIAMO INSIEME PER IL FUTURO}

È un dato di fatto che la pandemia globale da Covid-19 costituisca una problematica di cui occuparsi in maniera concreta e urgente ${ }^{43,44}$. I temi principali emersi dal nostro studio sono due: il peggioramento dello stile alimentare e l'aumento della FI.

Come abbiamo imparato dalla epidemia causata dalla SARS (Severe Acute Respiratory Syndrome) nel 20022004 , la quarantena obbligatoria può condurre a un'alta prevalenza di stress, che si può manifestare sotto forma di irritabilità ${ }^{45}$, iperfagia e alimentazione incontrollata, fenomeno definito dagli Autori anglosassoni come binge eating ${ }^{46}$. Lo stare a casa per periodi prolungati può facilitare il consumo di snack e cibi palatabili ed è spesso associato a una ridotta attività fisica. La paura del virus sconosciuto, il pericolo del contagio all'interno dei nuclei familiari, in particolare nei confronti delle popolazioni più fragili, il senso di incertezza e precarietà, hanno portato la popolazione a vivere un

\section{MESSAGGI CHIAVE}

$\square$ Per food insecurity si intende la mancanza di accesso continuativo a cibo adeguato da un punto di vista della quantità e della qualità per una vita attiva e sana.

$\square$ La sindrome respiratoria acuta severa da coronavirus-2 (SARS-CoV-2) e le relative misure di contenimento hanno causato drammatiche conseguenze sociali ed economiche anche in aree benestanti come il nostro Paese.

$\square$ La pandemia da Covid-19 ha comportato un aumento drammatico della FI e un peggioramento dello stile di vita alimentare nei bambini, associato a un aumento del consumo di cibo spazzatura. - La quarantena obbligatoria e l'impossibilità di trascorrere tempo all'aria aperta hanno causato un aumento ponderale in età pediatrica.

$\square$ Il calo ponderale, se pur raro, è stato osservato prevalentemente in età adolescenziale.

$\square \grave{E}$ di fondamentale importanza promuovere misure atte a supportare e a garantire la sicurezza alimentare e lo stile di vita sano.

lungo periodo di forte tensione psicofisica e sociale, contribuendo a variazioni dello stile dietetico, in una relazione bidirezionale tra tono dell'umore e alimentazione ${ }^{47}$.

In relazione alla FI, ci aspettiamo che la riduzione dell'accesso a cibo sufficiente e salutare, specie a danno di una popolazione in rapido accrescimento staturo-ponderale, psicologico ed emozionale quale quella pediatrica, sia ulteriore causa nel futuro dell'aumento delle disparità riguardo alle aspettative di salute. Il nostro studio supporta, come già affermato da numerosi Organismi internazionali, la necessità di agire quanto prima, affinché vengano adottate misure, non soltanto a effetto immediato ma in particolar modo a lungo termine ${ }^{48}$, per facilitare la distribuzione e la disponibilità dei costituenti di un'alimentazione sana e bilanciata e supportare le fasce di popolazione più fragili (e tra queste $\mathrm{i}$ bambini), garantendo un apporto di cibo adeguato per tutti ${ }^{49}$. 
Indirizzo per corrispondenza:

Egidio Candela

egidiocandela@gmail.com

\section{Bibliografia}

1. Nord M, Andrews MS, Carlson S. Household Food Security in the United States, 2008; Department of Agriculture, Economic Research Service: Washington, DC, USA, 2008.

2. Cook JT, Frank DA, Levenson SM, et al. Child food insecurity increases risks posed by household food insecurity to young children's health. J Nutr 2006;136(4):1073-6. doi: 10.1093/jn/136.4.1073.

3. Cook JT, Frank DA, Berkowitz C, et al. Food insecurity is associated with adverse health outcomes among human infants and toddlers. J Nutr 2004;134(6):1432-8. doi: 10. 1093/jn/134.6.\#\#.

4. Loopstra R, Reeves A, Stuckler D. Rising food insecurity in Europe. Lancet 2015;385 (9982):2041. doi: 10.1016/S0140-6736(15) 60983-7.

5. Loopstra R, Reeves A, McKee M, Stuckler D. Food insecurity and social protection in Europe: Quasi-natural experiment of Europe's great recessions 2004-2012. Prev Med 2016;89:44-50. doi: 10.1016/j.ypmed.2016. 05.010 .

6. Zaçe D, Di Pietro ML, Caprini F, de Waure $\mathrm{C}$, Ricciardi W. Prevalence and correlates of food insecurity among children in high-income European countries. A systematic review. Ann Ist Super Sanita 2020;56(1):90-8. doi: 10.4415/ANN_20_01_13.

7. European Union. European Commission Database. EUROSTAT. Available online: http://ec.europa.eu/eurostat/data/database (accessed on 18 March 2018).

8. Koo JR, Cook AR, Park M, et al. Interventions to mitigate early spread of SARS-CoV-2 in Singapore: A modelling study. Lancet Infect Dis 2020;20 (6):678-88. doi: 10.1016/ S1473-3099(20)30162-6.

9. Smith MD, Wesselbaum D. Covid-19, food insecurity and migration. J Nutr 2020;150 (11):2855-8. doi: $10.1093 /$ jn/nxaa270

10. Committee on World Food Security. High-Level Panel of Experts on Food Security and Nutrition (HLPE). Impact of Covid-19 on Food Security and Nutrition (FSN). Interim HLPE 2020;1:1-8.

11. Niles MT, Bertmann F, Belarmino EH, Wentworth T, Biehl E, Neff R. The early food insecurity impact of Covid-19. Nutrients 2020:12(7):2096. doi: 10.3390/nu12072096.

12. World Food Programme. Global Monitoring of School Meals During Covid-19 School Closures. 2020 (accessed on 16 November 2020).

13. Martini M, Gazzaniga V, Bragazzi NL, Barberis I. The Spanish influenza pandemic: a lesson from history 100 years after 1918 . J Prev Med Hyg 2019;60(1):E64-E67. doi: 10. 15167/2421-4248/jpmh2019.60.1.1205.

14. Althoff RR, Ametti M, Bertmann F. The role of food insecurity in developmental psychopathology. Prev Med 2016;92:106-109. doi: 10.1016/j.ypmed.2016.08.012.

15. Post JD. Famine, mortality, and epidemic disease in the process of modernization. Econ. Hist Rev 1976;29(1):14-37. doi: 10.1111/j.1468-0289.1976.tb00238.x.
16. Belanger MJ, Hill MA, Angelidi AM, Dalamaga M, Sowers JR, Mantzoros CS. Covid19 and disparities in nutrition and obesity. $\mathrm{N}$ Engl J Med 2020;383(11):e69. doi: 10.1056/ NEJMp2021264.

17. Razzoli M, Pearson C, Crow S, Bartolomucci A. Stress, overeating, and obesity: Insights from human studies and preclinical models. Neurosci Biobehav Rev 2017;76(Pt A):154-62. doi: 10.1016/j.neubiorev.2017. 01.026

18. I determinanti sociali della salute: che cosa sono e come influenzano la nostra salute? di A. Rinaldi e Maurizio Marceca. Riflessioni Sistemiche 16 giugno 2017:104-19.

19. Marmot M. La salute disuguale. La sfida di un mondo ingiusto. Roma: Il Pensiero Scientifico Editore, 2016.

20. Maciocco G. I determinanti della salute. Una nuova, originale cornice concettuale. Salute Internazionale. 25 gennaio 2009.

21. Costa G, Stroscia M, Zengarini N, Demaria $\mathrm{M}$ (a cura di). 40 anni di salute a Torino, spunti per leggere i bisogni e i risultati delle politiche. Milano: Inferenze, 2017.

22. Hager ER, Quigg AM, Black MM, et al. Development and validity of a 2 -item screen to identify families at risk for food insecurity. Pediatrics 2010;126(1):e26-32. doi: 10.1542/ peds.2009-3146.

23. The Lancet Global Health. Food insecurity will be the sting in the tail of Covid-19. Lancet Glob Health 2020;8(6):e737. doi: 10.1016/S2214-109X(20)30228-X.

24. Pérez-Escamilla R, Cunningham K, Moran VH. Covid-19 and maternal and child food and nutrition insecurity: a complex syndemic. Matern Child Nutr 2020;16(3):e13036. doi: $10.1111 / \mathrm{mcn} .13036$

25. World Food Program. Covid-19 Will Double Number of People Facing Food Crisis Unless Swift Action is Taken. 2020.

26. Eurostat. GDP per Capita in EU Regions. Regional GDP per Capita Ranged from 30\% to $263 \%$ of the EU Average in 2018.

27. GBD 2017 Italy Collaborators. Italy's health performance, 1990-2017: Findings from the Global Burden of Disease Study 2017. Lancet Public Health 2019;4(12):e645e657. doi: 10.1016/S2468-2667(19)30189-6.

28. Tarasuk V, Fafard St-Germain AA, Mitchell A. Geographic and socio-demographic predictors of household food insecurity in Canada, 2011-2012. BMC Public Health 2019;19(1):12. doi: 10.1186/s12889-018-63442 .

29. Dudek H, Myszkowska-Ryciak J. The Prevalence and Socio-Demographic Correlates of Food Insecurity in Poland. Int. J Environ Res Public Health 2020;17(17):6221. doi: $10.3390 /$ ijerph 17176221 .

30. Demoskopika. La performance sanitaria. Indice di Misurazione e Valutazione dei Sistemi Regionali Italiani. 2019. http:77www. quotidianosanita.it/allegati/allegato8783063. pdf.

31. Dondi A, Piccinno V, Morigi F, Sureshkumar S, Gori D, Lanari M. Food Insecurity and Major Diet-Related Morbidities in Migrating Children: A Systematic Review. Nutrients 2020;12(2):379. doi: $10.3390 /$ nu12020379.

32. Morales DX, Morales SA, Beltran TF. Racial/ethnic disparities in household food insecurity during the Covid-19 pandemic: a nationally representative study. J. Racial Ethn Health Disparities 2020;1-15. doi: 10.1007/ s40615-020-00892-7.
33. Adams EL, Caccavale LJ, Smith D, Bean MK. Food insecurity, the home food environment, and parent feeding practices in the era of Covid-19. Obesity 2020;28(11):2056-63. doi: 10.1002 /oby. 22996 .

34. Pietrobelli A, Pecoraro L, Ferruzzi A, et al. Effects of Covid-19 lockdown on lifestyle behaviors in children with obesity living in Verona, Italy: a longitudinal study. Obesity 2020;28(8):1382-5. doi: 10.1002/oby.22861.

35. Baysun Ş, Akar MN. Weight gain in children during the Covid-19 quarantine period. J Paediatr Child Health 2020;56(9):1487-1488. doi: $10.1111 /$ jpc. 15105

36. He M, Xian Y, Lv X, He J, Ren Y. Changes in body weight, physical activity, and lifestyle during the semi-lockdown period after the outbreak of Covid-19 in China: an online survey. Disaster Med Public Health Prep 2020;16. doi: $10.1017 /$ dmp.2020.237.

37. Sidor A, Rzymski P. Dietary Choices and Habits during Covid-19 Lockdown: Experience from Poland. Nutrients 2020;12(6):1657. doi: $10.3390 /$ nu12061657.

38. Barrea L, Pugliese G, Framondi L, et al. Does Sars-Cov-2 threaten our dreams? Effect of quarantine on sleep quality and body mass index. J Transl Med 2020;18(1):318. doi: 10.1186/s12967-020-02465-y.

39. Zachary Z, Brianna F, Brianna L, et al. Self-quarantine and weight gain related risk factors during the Covid-19 pandemic. Obes Res Clin Pract 2020;14(3):210-6. doi: 10 . 1016/j.orcp.2020.05.004

40. Michels N, Sioen I, Boone L, et al. Longitudinal association between child stress and lifestyle. Health Psychol 2015;34(1):40-50. doi: $10.1037 /$ hea0000108

41. Fernandez-Rio J, Cecchini JA, Mendez-Gimenez A, Carriedo A. Weight changes during the Covid-19 home confinement. Effects on psychosocial variables. Obes Res Clin Pract 2020;14(4):383-5. doi: 10.1016/j.orcp. 2020.07.006

42. Hoffmann S, Cortés-García L, Warschburger P. Weight/shape and muscularity concerns and emotional problems in adolescent boys and girls: A cross-lagged panel analysis. J Adolesc 2018;68:70-7. doi: 10.1016/j.adolescence.2018.07.005

43. Oliveira TC, Abranches MV, Lana RM. Food (in) security in Brazil in the context of the SARS-CoV-2 pandemic. Cad Saude Publica 2020;36(4):e00055220. doi: 10.1590/0102$311 X 00055220$

44. Torero M. Without food, there can be no exit from the pandemic. Nature 2020;580 (7805):588-9. doi: 10.1038/d41586-020-011813 .

45. Brooks SK, Webster RK, Smith LE, et al. The psychological impact of quarantine and how to reduce it: rapid review of the evidence. Lancet 2020;395(10227):912-20. doi: 10.1016/S0140-6736(20)30460-8

46. Razzoli M, Bartolomucci A. The Dichotomous Effect of Chronic Stress on Obesity. Trends Endocrinol. Metab. 2016, 27, 504-515. 47. Polivy J, Herman CP. Mental health and eating behaviours: A bi-directional relation. Can. J. Public Health 2005;27(7):504-15. doi: 10.1016/j.tem.2016.04.007

48. Tamburlini G. L'impatto della pandemia Covid-19 sulla salute globale dei bambini. Medico e Bambino 2020;39(10):629-32

49. Food and Agriculture Organization of the United Nations (FAO). Addressing the Impacts of Covid-19 in Food Crises (accessed on 19 December 2020). 